\title{
La innovación en Colombia. ¿Qué reto nos espera?
}

\section{Resumen}

Este trabajo se desarrolló con el fin de aportarle al lector un nuevo conocimiento en lo referente al tema de innovación empresarial, teniendo como principales objetivos dar a conocer información de primera línea y lograr que se analice el tema de la innovación en Colombia, para que el lector saque sus propias conclusiones en lo que tiene que ver con los avances del país en cuanto al tema en mención.

También se busca crear conciencia en lo referente a la fusión que debe hacerse entre empresa, Estado y universidades para poder afianzar la investigación y desarrollo (I y D) y lograr, como resultado, la generación de nuevo conocimiento que culmine en innovaciones empresariales.

Palabras claves: Innovación empresarial, Investigación y Desarrollo (I y D), transferencia tecnológica, plan de desarrollo.

\section{Abstract}

This work was developed to provide the reader with new knowledge on the subject of business innovation, having as objectives: to present a first-line and also make discussion of the topic of innovation in Colombia to develop conclusions own by the reader, you have to do with the progress of our country on the issue in question.

It also seeks to raise awareness regarding the merger to be made between company, state and universities to strengthen research and development $(R \& D)$ achieving results in the generation of new knowledge that will lead to business innovation.

Keywords: Business innovation, R \& D, technology transfer, development plan.

Recibido: 25 de abril de 2012

Aprobado: 01 de agosto de 2012

\section{Introducción}

Como es bien sabido, el constante y vertiginoso cambio tecnológico-científico por el cual atraviesa el mundo en pleno siglo XXI, con grandes y portentosos descubrimientos (en especial, en lo referente a las telecomunicaciones, las biotecnologías y el uso de materiales inteligentes, entre otras), ha hecho indispensable tomar conciencia sobre la adopción de nuevas herramientas para adecuarse a esas profundas transformaciones y cambios contantes, para poder, a su vez, estar a la vanguardia de los acontecimientos mundiales.
No solo se trata de estar actualizado: también se busca que Colombia comience a pensar de manera diferente en cuanto a querer surgir cada día más. Cabe recordar que siempre se debe estar a la vanguardia de las nuevas tecnologías o innovaciones, pues son fundamentales para no sucumbir y quedar relegados ante los demás países desarrollados, con el fin de no seguir con esa gran brecha que lleva al subdesarrollo, y por ende, terminar más debilitados en materia de bienestar y desarrollo.

\footnotetext{
${ }^{1}$ Administrador de empresas, Especialista en gerencia estratégica de mercadeo, Magíster en administración y estudiante de PhD en gestión. Actualmente líder del grupo de investigación Fénix categorizado en Colciencias y coordinador de la línea de investigación de Comportamiento y Desarrollo Organizacional articulado con la Maestría en Administración de Organizaciones de la UNAD. Con estudios de investigación en Innovación Empresarial. jose.castellanos@unad.edu.co. Bogotá (Colombia).
} 


\section{Métodos.}

El desarrollo de la investigación se enfoca en un tipo de estudio exploratorio, donde se hace una revisión bibliográfica de aquellos autores que han realizado estudios previos en cuanto a la innovación empresarial; por lo tanto, el método de investigación es bibliográfico documental porque el objetivo es hacer una indagación acerca de la innovación en Colombia.

\section{Concepto de innovación.}

Existen varios conceptos de innovación debido a que muchos autores se han dedicado a investigar sobre el tema. Entre ellos se pueden citar a J. Schumpeter, C. Freeman, M. Porter y G. Hamel, entre otros. En este escrito se detallarán aquellos que ayuden a encauzar el conocimiento, y que, a su vez, estén bien fundamentados en lo que hace referencia a Innovación Empresarial.

El primer concepto es el presentado por C. Freeman (1982) quien define la innovación como "la utilización de conocimiento nuevo para ofrecer un nuevo producto o servicio que desean los clientes." Lo anterior hace referencia a que el hombre debe utilizar todo su conocimiento para generar ideas innovadoras acordes con lo que necesitan los usuarios. Es decir, la innovación es igual a invención + comercialización. (Freeman, 1982)

Por otra parte, M. Porter (1990) define la innovación como "una nueva manera de hacer cosas (invención) que se comercializan." El mismo autor propone tal definición a partir de su modelo de competitividad y productividad; o sea, el autor parte del hecho de que existen ideas, conocimientos, tecnologías en continua transformación y productos creados. Lo importante es descubrir e inventar otras maneras de conocer el mundo, llegar a nuevos saberes, perfeccionar las tecnologías y transformar o crear nuevos productos altamente competitivos. Con lo expuesto se concluye que el proceso de innovación no se puede separar del contexto estratégico y competitivo de la empresa. (Porter, 1990)

EI SNIC (Sistema Nacional de Innovación Colombiano) concibe la innovación empresarial como "La disposición mental y la nueva forma de pensar a cerca de las estrategias y prácticas de los negocios." Moisés Wassermann, investigador de la Universidad Nacional, afirma que "la innovación es un paso que va un poco más allá de la tecnología; es no solamente generar un producto o servicio, sino también la capacidad de estar mejorando productos, mejorando servicios existentes, creando realmente ciertos productos nuevos para suplir la necesidad de las personas.' (Wassermann, 2004. Pp. 10)

Así pues, la innovación efectiva es la que contribuye al éxito en una empresa en cuanto a mercadeo y finanzas se refiere. También impacta positivamente en el capi- tal tecnológico acumulativo - (know-how $)^{2}$ que genera valor en la organización, así como en el desarrollo de capacidades de aprendizaje permanentes, al generar procesos dinámicos de investigación y aprendizaje, y así afectar positivamente la competitividad y la productividad de la producción.

La experiencia de las empresas colombianas exitosas en innovación muestra que la innovación se halla en el centro de las estrategias del crecimiento de los negocios, y los encargados de su estructura (organización) y de su puesta en marcha son la alta gerencia y las juntas directivas. De esta forma, la cultura organizacional se hace en una fuente de generación de nuevo conocimiento y de desarrollo de nuevas ideas novedosas para aplicarlas en pro de la conquista de nuevos mercados.

La gerencia moderna de las organizaciones está sustentada en la generación y el desarrollo de una mentalidad innovadora, enmarcada dentro del aprendizaje permanente y el apoyo a la creatividad. Ello, con el fin retroalimentar el crecimiento de la competitividad empresarial en el mediano y el largo plazos.

\section{Clases de innovación.}

Según el objeto que persiguen, los proyectos de innovación se clasifican en cuatro grupos: innovación en gestión; en proceso; en productos; en servicios tecnológicos y capacitación (Sistema Nacional de Innovación, 1998).

\section{-Innovación en gestión}

Son aquellos proyectos orientados a mejorar la productividad de las empresas colombianas mediante la adopción de técnicas de mejoramiento continuo, control de la calidad, organización del trabajo y mejoramiento gerencial.

\section{-Innovación en procesos}

Se trata de proyectos que buscan generar mejoras en los procesos productivos con el fin de incrementar la productividad, mejorar la calidad y buscar un manejo responsable y respetuoso en la relación producciónmedio ambiente.

\section{-Innovación en producto}

Son aquellos proyectos orientados al diseño y la elaboración de nuevos productos, con determinadas características que garanticen un exitoso posicionamiento en los mercados externo e interno, y que incrementen la competitividad en la producción.

\section{-Innovación en servicios tecnológicos y capacitación}

Son aquellos proyectos que buscan mejorar o introducir un servicio tecnológico, o capacitar personal, con el fin de desarrollar un mayor control sobre los procesos de producción y garantizar una permanente disposición al 
cambio y la búsqueda de la competitividad empresarial. Teniendo en cuenta a Getec (2005), en su libro Gestión de la innovación, esta también se puede clasificar así:

Según su impacto:

- Innovación radical

- Innovación incremental

Según el efecto:

- Innovación continuista

- Innovación rupturista

Según el origen de la innovación:

- Dirigida a la tecnología

- Impulsada por el mercado

Según la escala en que se realice el proceso de innovación:

- Programa-proyecto-operación

- Grupo empresarial-empresa-unidad de negocio

- Sector-mercado

- Regional-nacional-mundial

\section{Proyectos de Innovación en las Empresas.}

Uno de los principales apoyos brindados por el Gobierno al desarrollo de una capacidad empresarial para la innovación es financiar proyectos realizados directamente por unidades productivas. Los proyectos empresariales de innovación han sido financiados con la línea de crédito establecida entre Colciencias ${ }^{3}$, Bancoldex ${ }^{4}$ y el Fondo Nacional de Garantías.

\section{¿De dónde surge la innovación en Colombia?}

Sus primeras bases están determinadas por la Revolución Industrial, muchos de cuyos avances significaron un gran camino en los procesos industriales de la época, y donde estos se expandieron con gran rapidez alrededor del mundo, lo cual dió como resultado lo que hoy en día se llama producción en masa.

En general, esta primera visión de innovación tecnológica permite elevar los estándares de vida de la población y transformar las condiciones relacionadas con la forma de vida y de trabajo de las personas. Sin embargo, solo hasta los años cincuenta del siglo pasado, con autores como (Abramovitz, 1956) y (Solow, 1957) se empieza a reconocer la verdadera importancia que tienen la tecnología y la innovación en cuanto al crecimiento económico de un país.

Se debe tener en cuenta que antes de los años cincuenta del siglo pasado, país que fuera potencia económica se lo debía a su acumulación de riqueza. La tecnología y la innovación solo eran aportes ajenos a esto, no representaban mayor relevancia ante una eventual expansión de la producción y la economía.

Después de un largo trabajo desde esos mismos años cincuenta, solo hasta finales de los ochenta y comienzos de los noventa del siglo XX, se incorporó de forma definitiva el hecho de que la tecnología y la innovación son parte fundamental de la economía (Romer, 1990), y que estas surgen de nuevo conocimiento científico, que con conceptos avanzados en diseño, nuevos programas (software), automatización, descubrimientos médicos y biológicos, búsqueda y diseño de materiales inteligentes, y nuevos conceptos o estrategias en cuanto a mercadotecnia y servicios, entre otros, son la base para que se genere en la economía una amplia expansión de la producción en todas sus ramas, lo cual da como resultado un incremento en la producción, con nuevos bienes y servicios, y maximiza todas las herramientas alrededor de este nuevo conocimiento.

Con este nuevo avance, la acumulación de riqueza se hace evidente, dejando de lado que solo quienes tuvieran dinero guardado tendrían la clave del desarrollo económico. Según el mismo autor citado, "Para la teoría económica neoclásica, la explicación del crecimiento se centró en la acumulación de capital como la principal fuente endógena de expansión de la producción". (Romer, 1990. Pp. 15).

\section{¿Para dónde vamos?}

Según la Constitución de 1991, Colombia, como Estado Social y de Derecho, deberá proveer, en principio del gobierno, las herramientas necesarias para que la ciudadanía pueda ejecutar los planes de innovación que estén relacionados con el mejoramiento continuo de la población y de las normas que internacionalmente rigen al respecto.

Sabiendo que Colombia es un país de mucha riqueza agroindustrial, con potencia hídrica, con dos grandes puertos por el Pacífico y el Atlántico, queda como tarea sacar las fortalezas que tienen los colombianos y brindarlas al exterior, de manera que estas inversiones extranjeras se vean reflejadas en una mejor inversión educativa y cultural para la población.

Por otro lado, habrá que plantear metas a corto, mediano y largo plazo, que permitan dar frutos para el futuro del país. De esta manera, y a pesar de los inconvenientes económicos que viven (una larga brecha existente entre ricos y pobres y la falta de empleo, entre otras), no serán barreras para lograr los objetivos que plantea Visión Colombia 2019 (fundamentar el crecimiento y el desarrollo social de la ciencia, la tecnología y la innovación). 
Para ello se necesita el esfuerzo sin tregua de cada uno de los colombianos, por querer superarse cada día más y ser más competentes y comprometidos con los propios quehaceres diarios, siempre en procura de una mejor familia, una mejor sociedad y un mejor país.

Para llevar a cabo este tipo de esfuerzos Colombia ha adoptado una serie de planes y objetivos que se ven plasmados en el documento Visión Colombia 2019. En él se destaca como prioridad para ser tecnológicamente innovadores que el país deberá dar un gran paso a una educación donde la cobertura sea mayor, y donde, en lo posible se cubra en su totalidad a las personas laborando.

Con base en lo anterior, se tienen planteados los siguientes objetivos: ampliar y mejorar la cobertura estudiantil alrededor del país; generar mayor empleo; redefinir los programas o los contenidos educativos; poner al país a la vanguardia de la tecnología; explorar nuevos mercados aprovechando las bondades del país; generar confianza en países extranjeros para que inviertan en Colombia y traigan consigo su tecnología (Visión Colombia 2019, 2010).

Para lograr las metas propuestas se debe contar con una estrategia clara, y esta debe ser la unión del Gobierno, la empresa y la academia. Las universidades juegan un rol importante en tal proceso, pues son las encargadas de generar e impartir conocimiento, representado en la enseñanza, además de llevar los procesos de investigación, transferir el conocimiento y la tecnología, y algo muy importante: desarrollar una labor social conforme a las necesidades de la comunidad.

Prueba de lo anterior se enmarca dentro del Foro de Conocimiento Intellectus donde se trata el tema de la tercera misión de la universidad y se lo enfoca en el emprendimiento y la innovación, teniendo en cuenta la estrategia de I + D + I (investigación + desarrollo + innovación). Es un estudio realizado por la Unión Europea, y tuvo como meta para 2010 "construir la Europa del conocimiento y para el crecimiento" (Bueno, 2007).

Por otro lado, en el continente americano un ejemplo de cómo dar pasos hacia la I y D (investigación y desarroIlo) se refleja en los Estados Unidos. Este país pone al servicio de la I y D un aproximado de US \$ 85 a 90 mil millones de dólares, equivalentes a un punto porcentual del PIB (producto interno bruto); también forman parte de este proceso las instituciones privadas o filantrópicas que están estrechamente relacionadas con los laboratorios de I y D, los cuales con sus aportes, ayudan en su desarrollo. Estas ayudas económicas que proveen a los laboratorios de investigación hacen posible el crecimiento como potencia mundial del país.

Además Estados Unidos cuenta con un innovador sistema de educación, diseñado para que las instituciones educativas participen de manera activa en el desarrollo de investigaciones y tecnologías nuevas, y donde la participación de los docentes en los laboratorios de I y $\mathrm{D}$ permite obtener de patentes para las universidades, y así generar los recursos financieros para seguir trabajando en I y D y, a su vez, crear innovaciones.

Volviendo al caso de Colombia, el país debe apoyarse en organismos o programas internacionales que permitan una visión más global de lo que en realidad se pretende con la innovación. Los Manuales de Frascati, Oslo, Camberra, y más recientemente, el de Bogotá, muestran verdaderos indicios al respecto desde el punto de vista de la creación de indicadores para medir la innovación; en especial, el Manual de Bogotá que se enfoca en la verdadera problemática de las empresas latinoamericanas.

Según el manual de Frascati (OCDE, 1993), la I y D incluye el desarrollo creativo llevado a cabo de forma sistemática para incrementar el volumen de los conocimientos humanos, culturales y sociales, y el uso de estos conocimientos para derivar en nuevas aplicaciones. En ese orden de ideas, es claro que la invencióninnovación se alimenta del conocimiento resultante de la experiencia acumulativa de las actividades de I y $\mathrm{D}$.

Según en el Manual de Oslo (OCDE, 1997:10), las actividades de innovación de producto-proceso (TPP), se definen como todos aquellos pasos científicos, organizacionales, financieros y comerciales orientados a la implementación de productos o procesos nuevos o tecnológicamente mejorados. El Libro Verde de la Innovación (Comisión Europea, CE, 1995), por ejemplo, considera la innovación como la fuerza motriz que impulsa a las empresas hacia objetivos ambiciosos a largo plazo, y la que conduce a la renovación de las estructuras industriales y a la aparición de nuevos sectores de la actividad económica.

En fin son innumerables las historias que dan luces sobre cómo se puede hacer I y $D$ en los países cuando se quiere y se cuenta con los recursos necesarios, como ocurre en los países industrializados. Colombia tendrá que lidiar con una limitada economía, un desempleo alto y un sistema educativo muy complejo en cuanto al desarrollo de I y D se refiere; sin embargo, no todo podría salir mal, pues lo que se debe hacer como país rico en agro y en recursos naturales, es aprovechar las propias fortalezas y virtudes, unirlas en un todo para el bien común, que genere un sistema de indicadores de I y D, capaz de mostrar según estándares internacionales, que para ser un país en vías de ser tecnológicamente innovador se debe contar para mediados de 2019 con una participación de alrededor del $0,1 \%$ de la población inmersa en investigación, y con, aproximadamente, 20 centros de investigación. 


\section{Su importancia}

La recuperación de la inversión tras un desarrollo tecnológico, según Thurow (2000) en su libro «Construir riqueza", se divide así: la tasa de rentabilidad que se obtiene del gasto en I y D está alrededor de un $24 \%$, mientras que los beneficios sociales a través de la rentabilidad en cuanto a retorno se refiere son de alrededor de más del $65 \%$.

Dentro de los principales autores que han investigado acerca del tema, se destaca el trabajo de Porter (1990), quien divide las actividades de una empresa en un conjunto de áreas funcionales cuya razón de ser es la creación de valor, y las cuales corresponden a: logística interna, operaciones o producción, logística externa, mercadotecnia y ventas y servicios.

La tecnología se encuentra involucrada en cada actividad desarrollada por la empresa; luego, el cambio tecnológico puede afectar la competencia a través de su impacto sobre cualquier actividad (Porter, 1990. Pp, 167).

En este orden de ideas, la ventaja competitiva es una función de la eficiencia lograda por las compañías para administrar un sistema de mayor valor e incorporarlo al producto final ofrecido, en lo que es determinante la diferenciación (Porter, 1990. Pp, 40). Esta idea se puede reflejar en lo siguiente:

Para obtener una ventaja competitiva sobre la competencia, una compañía ha de ser capaz de proveer un valor al cliente comparable al de sus competidores, pero a la vez ha de realizar sus actividades de una manera más eficiente que éstos (menor costo), o desarrollar actividades de una manera única en la que se cree un mayor valor al cliente que permita percibir una recompensa adicional en términos de precio (premium price) para la compañía (diferenciación). (Porter, 1990. Pp, 40).

\section{¿Qué papel juegan el Gobierno, las empre- sas y la educación?}

Como anteriormente se describió, hay que aprovechar las fortalezas por las cuales el país es potencia mundial. Como se sabe, las grandes riquezas naturales de Colombia y el potencial en cuanto al agro que el país debería saber aprovechar y explotar, además del posicionamiento marítimo, son algunas de las innumerables posibilidades por las cuales el Gobierno deberá emprender con mayor fuerza la unión con los demás países, como ya lo ha hecho siendo integrante del comercio con diferentes regiones, e impulsando las relaciones con nuevos países y mercados. Además se deben consolidar las relaciones con la Comunidad Europea y los países asiáticos, para así generar un fortín de comercio bilateral cada vez más fuerte.
Por tanto, las empresas y la comunidad educativa requieren que sus esfuerzos sean más loables; es decir, que estas dos partes lleguen a un mejor acuerdo de cohesión, donde la empresa ayude con fondos a las instituciones educativas, para que estas generen I y D, y, por otro lado, la comunidad educativa devuelva a las empresas ese conocimiento científico, tecnológico e innovador en herramientas, que les permita tanto competir como permanecer en los mercados internos y externos, con calidad, servicio y un rendimiento económico sostenible a través del tiempo, mejorando día tras día su producto, su mercado y su servicio.

Entre tanto, el papel del Estado es supremamente importante, en la medida en que la inversión que este deposite hacia el sistema de investigación e innovación, sea capaz de sostener los desarrollos de I y D + I (investigación y desarrollo + innovación).

Muy a pesar de esta gran inversión, cabe recordar también que los beneficios económicos y sociales son muy cuantiosos y benéficos para las partes involucradas, pues como no sobra recalcar, esto no será posible sin la unión de estamentos privados, el gobierno y entidades educativas y empresariales.

Es decir, la verdadera innovación colombiana ha de implicar grandes y profundos conocimientos, infraestructura, equipos y capacidades; por ejemplo, un recurso tecnológico (tecnología, equipo o know-how), un usuario (Pymes, gran industria, entidad pública, entre otros.), un intermediario (laboratorio, centro de I y D, entre otros), cuya función es vincular a los dos primeros. Además de la interacción entre sus elementos, la transferencia de tecnología (TT) lleva implícitas consideraciones en relación con patentes, Copyrights, derechos de autor y registro de marcas.

\section{Conclusiones}

Si bien a lo largo de las próximas décadas las nuevas tecnologías e innovaciones estarán dadas en los campos de la nanotecnología, la búsqueda de nuevos materiales inteligentes, nuevo software, nuevos elementos biotecnológicos y la búsqueda incesante de nuevos combustibles amigables con el medio ambiente, es también cierto que todo apunta a crear elementos desmaterializándolos; es decir, a crear con mayores beneficios con menos material; también lo es la integración virtual o informática, pues todo será manejado en torno a las computadoras. Así se logrará viajar alrededor del mundo sin salir nunca de la casa o de la oficina, lo que se llamaría virtualidad total.

El Gobierno debe adoptar el papel de líder acercando a la academia, la empresa y la sociedad, para que todas desarrollen los mismos objetivos en cuanto a innovación empresarial se refiere, y trazados en el Plan Vi- 
sión Colombia 2019 expuesto en este mismo artículo. La tarea no queda completa si no desarrollan planes de divulgación en las micro, las pequeñas, las medianas y las grandes empresas. Para que este objetivo se cumpla las organizaciones se deben agrupar en clusters según su misión afín.

El Estado debe preocuparse por el bienestar del Sistema Nacional de Innovación. Este organismo debe asumir el rol de creador de obras sociales, para facilitarles la labor a las organizaciones pertenecientes al sistema, como, por ejemplo, la implementación de vías de comunicación, de puertos marítimos y fluviales con la suficiente capacidad para la región, y de servicios de primera necesidad (agua, energía, teléfono, TICs, entre otros). También debe funcionar como pilar de leyes que fomenten el desarrollo de la región, enfocado en las Políticas Públicas.

Las universidades deben desarrollar dos funciones fundamentales dentro del ambiente de un Sistema de Innovación Empresarial: la transmisión del conocimiento enfocado en prácticas empresariales que deben estar interconectadas con las necesidades del medio ambiente y el monitoreo de todas las actividades del sistema. Esto se debe hacer según el enfoque de la investigación e interconexión empresarial.

El Sistema Nacional de Innovación debe poseer un enfoque sistémico y estar en pos de descubrir nuevas oportunidades en los mercados. No debe enfocarse solo en los mercados nacionales sino que debe entender que ya no existen fronteras y el mercado también se mueve en torno a los bloques económicos.

El manejo de redes y tecnología, al igual que la innovación y la I y $\mathrm{D}$, son elementos clave que posee el líder emprendedor de hoy en día, y que definen con claridad su éxito o su fracaso en los proyectos.

Por último, se debe fomentar una cultura innovadora en todos los elementos que hacen parte del Sistema Nacional de Innovación, y esta debe ser implementada en todas las organizaciones por parte de las altas directivas, para que se fomente la creatividad y se obtengan como resultado sistemas de innovación que, a su vez, se traduzcan en ventajas competitivas para las entidades.

\section{Referencias bibliográficas}

Abramovitz, M. (1956). Catching Up, Forging Ahead and Falling Behind. Pp. 46.

Bueno, E. (2007) Gestión del Conocimiento en Universidades y Organismos Públicos de Investigación. Madrid: Universidad Autónoma de Madrid.

Comisión Europea (1995) El Libro Verde de la Innovación.
Dinero (Junio 8, 2001) Biotecnología: Apuesta del Milenio. No. 134. Pp 32-46.

Drucker, P. (1986) La Innovación y el empresario Innovador. Buenos Aires: Ed. Norma

Freeman, C. (1997). The Economics of Industrial Innovation. [Versión electrónica] Disponible en: http://books. google.com.co/books/about/The_Economics_of_Industrial_Innovation.html?id=XTxkwzP1xu0C\&redir_esc=y. Recuperado el 28 de julio de 2011. Pp 12.

Freeman, C. (1982). Unemployment and Technical Innovation: a Study of Long Waves in economic Development. Frances Pinter

Garrido, C. (2005) Collection B. La Universidad en la Sociedad del conocimiento. Universidad Virtual de México: innovación y aprendizaje tecnológico [Versión electrónica] Disponible en: http://e-spacio.uned.es/fez/view. php?pid=bibliuned:19377. Recuperado el 28 de julio de 2011. Pp 6-8.

Hamel, Gary (2002) Leading the Revolution: How to Thrive in Turbulent Times by Making Innovation a Way of Life. Printed in the United States of America: Harvard Business School.

Jaimes, A. (2007). La Gestión del Conocimiento en entidades de Conocimiento: El Caso de los Laboratorios Académicos y de las Empresas de Base Tecnológica en Europa. Barranquilla: Universidad del Norte. Pp 168 -190 .

Jaramillo, H. (2001) Normalización de Indicadores de Innovación Tecnológica en América Latina y el Caribe - Manual de Bogotá. Bogotá: Red Iberoamericana de Indicadores de Ciencia y Tecnología / Organización de Estados Americanos - OEA / COLCIENCIAS.

Keller, W. (1996), Absorptive Capacity: On the Creation and Acquisition of Technology in Development. Journal of Development Economics 49: Pp. 199-227.

Kim Chan, W. Mauborgne, Renée (2005) La Estrategia del Océano Azul. Cómo desarrollar un nuevo mercado donde la competencia no tiene ninguna importancia. Harvard Business School Publishing Corporation.

Laverde R., Jairo César (2000) Plan Estratégico del Programa Nacional de Desarrollo Tecnológico Industrial y Calidad 2000-2010. Bogotá: Sistema Nacional de Innovación.

Mojica, Francisco (2008). Forecasting y Prospectiva: dos alternativas complementarias para adelantarnos al futuro. Bogotá: Universidad Externado de Colombia.

Nonaka, I. Takeuchi, H. (1999). La Organización Creadora de Conocimiento: Cómo las compañías japonesas crean la dinámica de la innovación. Cap.3. Pp. México: 88 - 94. Oxford University Press. 
OCDE (1993). Organización para la Cooperación y el Desarrollo Económico. Paris. Frascati Manual.

Oslo, Manual (1997). The Measurement of Scientific and Technological Activities. Proposed Guidelines jor Collecting and interpreting Innovation Data. Madrid: European Commission, Eurostat.

Plan Visión Colombia, II centenario 2019. Bogotá: Departamento Nacional de Planeación. Dirección de Desarrollo Urbano y Política Ambiental.

Porter, M. (1990). The Competitive Advantage of Nations. New York: The Free Press. A Division of Macmillan, Inc. Pp. 40.

Porter, M. (1985). The Competitive Advantage of Nations. New York: The Free Press. A Division of Macmillan, Inc. Pp. 167.

Romer, P. (1990). "Endogenous Technological Change," Journal of Political Economy Pp. 15.

Sala-i-Martin, X. (2011). The Global Competitiveness Report 2010 - 2011. World Economic Forum. Geneva: Published by the World Economic Forum within the framework of the Centre for Global Competitiveness and Perfomance. Pp. 32 - 37.

Sistema Nacional de Innovación. (1998). Cartilla del Instituto Colombiano Para el Desarrollo de la Ciencia y la Tecnología Francisco José de Caldas- COLCIENCIAS. Nuevo escenario de la competitividad. Bogotá, septiembre. Pp. 24.
Solow, R. (1957), Technical Change and the Aggregate Production Function, en Review of Economics and Statistics, $N^{\circ} 39$, Cambridge, pp. 318-320.

Thurow, L. (2000). Construir riqueza. Editorial Vergara. Argentina.

Truffer, I. No. 24 (mayo de 2002). Ciencia, Tecnología y Sociedad. Evaluación de las actividades científico tecnológicas. [Versión electrónica] Disponible en: http://www.revistacdyt.uner.edu.ar/articulos/descargas/ cdt24_truffer.htm Recuperado el 02 de agosto de 2011. Pp 3-7.

Wassermann, M. (2004). Revista Javeriana. La Visión de los Empresarios, La Competitividad e Innovación de las Pymes. Pp. 10-16.

\section{Notas}

${ }^{2}$ Terminología inglesa que traduce saber hacer o cómo hacer y que hace referencia al conjunto de conocimientos que se adquieren con la experiencia, el aprendizaje y la información y que no están protegidos por una patente, por lo cual el único medio de protección para la empresa es el secreto de su fórmula el cual, finalmente, es lo que le da ventaja competitiva y el éxito comercial a la compañía. También cabe mencionar que puede ser el conocimiento de las técnicas necesarias para ejecutar un proceso productivo y que se mantiene en reserva.

${ }^{3}$ Departamento Administrativo de Ciencia, Tecnología e Innovación.

${ }^{4}$ Banco de Comercio Exterior de Colombia S.A. 